\title{
An analysis of the expression and function of myeloid differentiation factor 88 in human osteosarcoma
}

\author{
$\mathrm{JUN} \mathrm{CHEN}^{1 *}, \mathrm{JIAN} \mathrm{HE}^{1^{*}}, \mathrm{YUE} \mathrm{YANG}^{2 *}$ and JIANNONG JIANG ${ }^{1}$ \\ ${ }^{1}$ Department of Orthopedics, The Affiliated Yixing Hospital of Jiangsu University, Yixing, Jiangsu 214200; \\ ${ }^{2}$ Department of Surgery, The Third Affiliated Hospital of Nanjing University of Chinese Medicine, \\ Nanjing, Jiangsu 210001, P.R. China
}

Received July 26, 2016; Accepted July 27, 2017

DOI: $10.3892 / 01.2018 .9297$

\begin{abstract}
The aim of the present study was to investigate the expression and function of myeloid differentiation factor 88 (MyD88) in osteosarcoma. Immunohistochemical staining was used to detect MyD88 protein in osteosarcoma tissues and matched normal bone tissues. The association between MyD88 expression and the clinical characteristics of patients with osteosarcoma was analyzed. Furthermore, survival analysis of patients with osteosarcoma was performed to study the association between MyD88 expression and patient prognosis. Finally, the effect of the MyD88 inhibitor, ST2825, on the proliferation and apoptosis of the human osteosarcoma cell line U2OS was examined. Additionally, cell proliferation, invasion and apoptosis were examined using an MTT assay, Transwell assay and Annexin V-fluorescein isothiocyanate staining kit, respectively. The expression of proteins associated with the $\mathrm{NF}-\kappa \mathrm{B}$ signaling pathway was analyzed by western blotting. The positive expression rate of MyD88 in osteosarcoma and normal bone tissues was 71.4 and $6.1 \%$, respectively. Statistical analysis demonstrated that MyD88 was not associated with gender, age, histological type or tumor location, but that it was associated with Enneking stage and tumor metastasis $(\mathrm{P}<0.05)$. According to the survival analysis, patients with osteosarcoma in the high MyD88 expression group displayed a reduced overall survival rate $(\mathrm{P}<0.05)$. Furthermore, inhibition of MyD88 by ST2825 in U2OS cells resulted in a marked decrease in cellular proliferation and migration, and an increase in the rate of apoptosis $(\mathrm{P}<0.05)$. Notably, ST2825 significantly decreased cyclin D1, matrix metallopeptidase-9 and nucleus p65 expression, but increased
\end{abstract}

Correspondence to: Dr Jiannong Jiang, Department of Orthopedics, The Affiliated Yixing Hospital of Jiangsu University, 75 Tongzhenguan Road, Yixing, Jiangsu 214200, P.R. China E-mail: jiannongjiang@126.com

${ }^{*}$ Contributed equally

Key words: osteosarcoma, myeloid differentiation factor 88, ST2825, proliferation, migration, apoptosis cleaved-caspase 3 expression in ST2825-treated U2OS cells $(\mathrm{P}<0.05)$. The results of the present study indicated that MyD88 expression is associated with the progression of osteosarcoma and may be a potential therapeutic target for the treatment of osteosarcoma.

\section{Introduction}

Osteosarcoma, which frequently occurs in teenagers, is the most common form of primary malignant bone tumor, with a high incidence of fatality (1). The specific molecular and pathological mechanisms underlying the development of osteosarcoma have not been fully elucidated. Therefore, there remains a lack of specific therapies for the clinical treatment of this disease (2). Thus, there is an urgent requirement to study the pathological mechanisms and to develop targeted drugs so as to improve the prognosis of patients with osteosarcoma. Myeloid differentiation factor 88 (MyD88) has been demonstrated to contribute to the occurrence and development of multiple types of tumors, including breast (3), ovarian (4), liver (5), pancreatic and colon $(6,7)$ cancer. However, there has been a distinct lack of previous research focusing on the expression and function of MyD88 in osteosarcoma tissue.

Upon activation of toll-like or interleukin (IL)-1 $\beta$ receptors, MyD88 activates a signaling cascade that culminates in the activation of nuclear factor- $\kappa \mathrm{B}(\mathrm{NF}-\kappa \mathrm{B})$ and the expression of proinflammatory genes. $\mathrm{NF}-\kappa \mathrm{B}$ is an important modulator in tumor progression (8). Activation of $\mathrm{NF}-\kappa \mathrm{B}$ contributes to cancer metastasis by inducing expression of metastasis-associated proteins. A previous study found that patients whose osteosarcoma had active NF- $\kappa \mathrm{B}$ experienced shorter median overall survival time compared with those patients whose osteosarcoma had inactive $\mathrm{NF}-\kappa \mathrm{B}$ (9). Inhibitors of $\mathrm{NF}-\kappa \mathrm{B}$ signaling suppressed expression of NF- $\mathrm{B}$-modulated metastasis-associated proteins and led to blockage of the metastatic mechanism in osteosarcoma cells, in vitro and in vivo $(10,11)$. Based on these observations, we hypothesized that MyD88 may serve a key role in osteosarcoma pathogenesis and development. A specific inhibitor of MyD88, ST2825, is a type of halogenated heptapeptide, which inhibits the dimerization of MyD88 via competitive binding to the Toll interleukin-1 receptor (TIR) domain of MyD88, thereby blocking the Toll-like receptors/IL signaling pathway and inhibiting the activation of NF- $\mathrm{KB}(11)$. 
The aim of the present study was to elucidate the expression and function of MyD88 in patients with osteosarcoma in association with their clinicopathological aspects. In addition, in the osteosarcoma U2OS cell line, the potential use of a MyD88 inhibitor, ST2825, was assessed as a novel drug targeting molecular pathways involved in the progression of osteosarcoma.

\section{Materials and methods}

Patient information. A total of 98 patients with postoperatively pathologically confirmed osteosarcoma following resection were selected and enrolled into the present study at the Affiliated Yixing Hospital of Jiangsu University (Yixing, China) between June 2001 and February 2010. During surgery, osteosarcoma tissues and matched normal peritumoral bone tissues (control) were sampled. Prior to the study, the research was approved by the by the Ethics Committee of the Affiliated Yixing Hospital of Jiangsu University, and written informed consent was provided by all patients or by an appropriate guardian. Patient information was collected throughout the study, including patient's name, age, anatomical site, histological subtype, metastasis and Enneking grade (12). Exclusion criteria included patients who had received radiotherapy or chemotherapy prior to surgery. All patients were followed up for a 5 -year period, either by telephone or by regular hospital visits, every 3 months.

Immunohistochemistry $(\mathrm{IHC})$ and hematoxylin and eosin $(H \& E)$ staining. All fresh specimens incised during the surgery were fixed in $10 \%$ formalin for $24 \mathrm{~h}$ at $4^{\circ} \mathrm{C}$. Following conventional paraffin embedding, tissue samples were sectioned at a thickness of $3 \mu \mathrm{m}$, and dried in the oven at $60^{\circ} \mathrm{C}$ for $4 \mathrm{~h}$. Sections were dewaxed in xylene and rehydrated in a series of anhydrous ethanol. Tissue sections were washed in PBS, 3 times for 5 min each time. Antigen retrieval was performed by placing tissue sections in sodium citrate buffer and heating in a pressure cooker, until steam was produced. The tissues were heated for a further $2 \mathrm{~min}$ from when the pressure cooker let off steam, and were then cooled naturally to room temperature. The sections were washed in distilled water twice and in PBS twice, for 3 min each time. Endogenous peroxidase activity was blocked by incubation of slides in peroxidase blocking solution (3\% hydrogen peroxide) at room temperature for $10 \mathrm{~min}$. Slides were washed in PBS 3 times, for $3 \mathrm{~min}$ each time. Non-specific binding was blocked by incubation of slides in 5\% normal rabbit serum (Zhongshan Golden Bridge Biotechnology, Co., Ltd., Beijing, China) at room temperature for $10 \mathrm{~min}$. Slides were then incubated with the primary antibody against MyD88 (1:50 dilution; catalog no. sc-11356; Santa Cruz Biotechnology, Inc., Dallas, TX, USA) at room temperature for $60 \mathrm{~min}$, and PBS replaced the primary antibody as the negative control. Slides were washed in PBS, 3 times, for 3 min each time. An appropriate biotin-labeled goat-anti-rabbit IgG secondary antibody (1:50 dilution; catalog no. DS-0002; Zhongshan Golden Bridge Biotechnology, Co., Ltd.) was titrated and the sections were incubated at room temperature for $10 \mathrm{~min}$. Slides were washed in PBS, 3 times, for 3 min each time. Streptavidin-peroxidase solution (Zhongshan Golden Bridge Biotechnology, Co., Ltd; goat-anti-rabbit IgG) was titrated and sections were incubated at room temperature for $10 \mathrm{~min}$. Slides were washed in PBS, 3 times, for 3 min each time, and stained with 3,3'-diaminobenzidine for $3 \mathrm{~min}$ for antigen detection. Sections were counterstained with hematoxylin, prior to dehydration with a series of alcohols (70\% ethanol, 95\% ethanol and absolute ethanol), and mounted with neutral balsam. H\&E staining was performed according to the standard protocols (13).

IHC analysis. IHC results were interpreted by three experienced pathologists in the Affiliated Yixing Hospital of Jiangsu University, and the specific methods of analysis used were performed a previously described (14). The double-blind method was adopted to count 15 fields of view for each section at $\mathrm{x} 400$ magnification with an optical microscope (Olympus, Tokyo, Japan). The positive cell rate referred to the average number of cells with positive MyD88 staining/per 100 cells. Interpretation of positive IHC results was performed following methods as previously described (5), according to the certain aspects. The staining intensity of positive cells was graded as follows: Negative staining, 0; pale yellow staining, 1; yellow staining, 2; and tan staining, 3 . The intensity of staining results were defined as follows: $0-2$, negative staining; or 3, positive staining. The percentage of positive cells was as follows: $0-5 \%$, negative expression; 6-25\%, weakly positive expression; $26-75 \%$, moderately positive expression; and $>76 \%$, strongly positive expression. Weakly, moderately and strongly positive expression was defined as positive staining.

Cell culture and grouping. The human osteosarcoma U2OS cell line was provided by the Cell Bank of the Chinese Academy of Sciences (Shanghai, China) and cultured in Dulbecco's modified Eagle's medium (DMEM; Gibco; Thermo Fisher Scientific, Inc., Waltham, MA, USA) containing 10\% fetal bovine serum (FBS; Sigma-Aldrich; Merck KGaA, Darmstadt, Germany) in an incubator with $5 \% \mathrm{CO}_{2}$ at $37^{\circ} \mathrm{C}$.

Groups. U2OS cells at the logarithmic stage were selected for the subsequent experiments. The MyD88 inhibitor, ST2825, was purchased from MedChemexpress LLC (Princeton, NJ, USA). ST2825 was firstly dissolved in dimethyl sulfoxide (DMSO), which was further diluted to 15 and $30 \mu \mathrm{M}$ with culture medium prior to each experiment. The cells were divided into four groups: The blank control group, the solvent (DMSO) treatment group, the low-dose ST2825 treatment group $(15 \mu \mathrm{M})$ and the high-dose ST2825 treatment group $(30 \mu \mathrm{M})$.

MTT assay for analysis of cell proliferation. MTT and DMSO were obtained from Solarbio Science and Technology Co., Ltd. (Beijing, China). Cells cultured with ST2825 (15 or $30 \mu \mathrm{M})$ were placed in a humidified incubator and maintained at $37^{\circ} \mathrm{C}$ with 5\% $\mathrm{CO}_{2}$ for 24,48 and $72 \mathrm{~h}$. A total of $20 \mu \mathrm{l}$ MTT solution was added to each well and maintained for a further $4 \mathrm{~h}$, prior to the termination of culture. DMSO (100 $\mu \mathrm{l}$; Sigma-Aldrich; Merck KGaA) was added to dissolve the formazan crystals. An enzyme immunoassay analyzer (Bio-Rad Laboratories, Inc. Hercules, CA, USA) was used to measure the optical density (OD) values at a wavelength of $490 \mathrm{~nm}$. 
Cell apoptosis analysis. An Annexin V-fluorescein isothiocyanate (FITC) staining kit (BioVision, Inc., Milpitas, CA, USA) was used to detect apoptosis in human U2OS cells, according to the manufacturers protocol. At 24, 48 and $72 \mathrm{~h}$, cells were digested by $0.25 \%$ pancreatin (Gibco; Thermo Fisher Scientific, Inc.) and washed by PBS twice, followed by centrifugation at 2,000 x $\mathrm{g}$ for $5 \mathrm{~min}$. Cells were washed in PBS and $1 \mathrm{X}$ binding buffer was added. A dual staining method was used and U2OS cells were labeled with $5 \mu \mathrm{l}$ Annexin V-FITC and $10 \mu \mathrm{l}$ propidium iodide, added to each well.

Transwell chamber migration assay. A cell migration assay was performed using 24-well Transwell chambers $(8-\mu \mathrm{m}$ pore size; Merck KGaA), according to the manufacturer's instructions.

Briefly, the cells were seeded at a density of $5 \times 10^{4} / 400 \mu \mathrm{l}$, and treated with ST2825 or vehicle (DMSO) in DMEM, place into the upper chambers of the Transwell assay. Fresh DMEM (600 $\mu \mathrm{l} /$ well) containing $20 \% \mathrm{FBS}$, was added to the bottom chambers. Following $24 \mathrm{~h}$ of culture, cells that migrated to the underside of the filter were fixed with methanol and stained with crystal violet for $1 \mathrm{~min}$ at room temperature, and counted using bright field microscopy. Each experiment was repeated on three independent occasions.

Western blot analysis. Subsequent to washing twice with PBS, the osteosarcoma cells treated for $48 \mathrm{~h}$ were centrifuged at $12,000 \mathrm{x}$ g for $15 \mathrm{~min}$ at $4^{\circ} \mathrm{C}$. A total cellular protein extraction kit (Bi Yuntian Biological Technology Institution, Nantong, China) and nucleoprotein extraction reagents (Bi Yuntian Biological Technology Institution, Nantong, China) were used to extract nucleoprotein according to the manufacturer's protocols. Total protein concentrations were quantified using the BCA method. Protein samples $(30 \mu \mathrm{g})$ were loaded and separated by $10 \%$ SDS-PAGE and transferred to polyvinylidene difluoride membranes. The membranes were blocked with 5\% non-fat milk (Beyotime Institute of Biotechnology) in TBST [20 mM Tris, $\mathrm{HCl}$ ( $\mathrm{pH}$ 7.2), $150 \mathrm{mM} \mathrm{NaCl}$ and $0.05 \%$ Tween-20] for $1 \mathrm{~h}$ at room temperature. The membranes were incubated with primary antibodies Histone 3 (H3) rabbit monoclonal antibody (mAb) (catalog no. 4499; 1:1,000 dilution), cyclin D1 rabbit mAb (catalog no. 2978; 1:1,000 dilution), p65 rabbit mAb (catalog no. $8242 ; 1: 1,000$ dilution), cleaved-caspase-3 rabbit $\mathrm{mAb}$ (catalog no. 9664; 1:1,000 dilution) and $\beta$-actin rabbit $\mathrm{mAb}$ (catalog no. $4970 ; 1: 1,000$ dilution) at $4^{\circ} \mathrm{C}$ overnight. All the primary antibodies were purchased from Cell Signaling Technology, Inc. (Danvers, MA, USA). The membranes were then washed and incubated with 5\% milk-diluted horseradish peroxidase-conjugated goat-anti-rabbit secondary antibody (catalog no. 7074; 1:5,000 dilution; Cell Signaling Technology, Inc.) for $1 \mathrm{~h}$ at room temperature. Membranes were washed again and proteins were detected using enhanced chemiluminescence reagent (Pierce; Thermo Fisher Scientific, Inc.). Images were captured on a gel imager (Tanon-3500 digital gel imaging system; Tanon Science and Technology Co., Ltd., Shanghai, China) and ImageJ (National Institutes of Health, Bethesda, MD, USA) was used for band densitometry analysis.

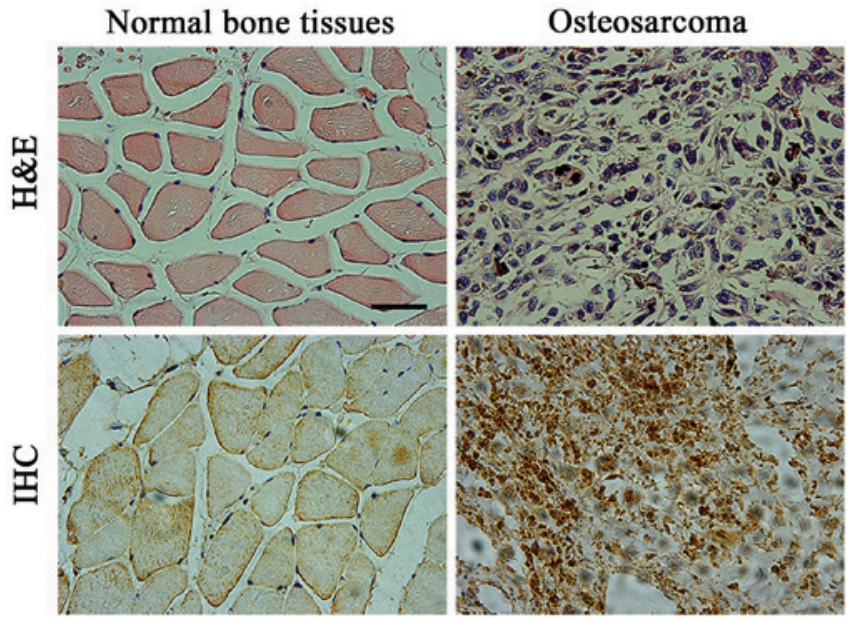

Figure 1. MyD88 expression in osteosarcoma tissue was increased significantly. Upper panel for H\&E staining results and lower panel for IHC staining results of MyD88. Magnification, x 400. H\&E, hematoxylin and eosin; IHC, immunohistochemistry; MyD88, myeloid differentiation factor 88.

Statistical analysis. Statistical analysis was conducted using SPSS, v.19.0 software (SPSS, IBM Corp, Armonk, NY, USA). Data are expressed as the mean \pm standard deviation. A $\chi^{2}$ test was used to analyze enumeration data, Kaplan-Meier method was used for survival analysis and the one-way ANOVA test was used to compare quantitative data. $\mathrm{P}<0.05$ was considered to indicate a statistically significant difference.

\section{Results}

$H \& E$ and IHC staining results of osteosarcoma and normal peritumoral bone tissues. Osteosarcoma specimens used in the present study were confirmed by postoperative pathological diagnosis. Typical H\&E staining results of osteosarcoma are demonstrated in Fig. 1. Osteosarcoma cells were mixed with trabecular or patchy osteoid tissues, and osteosarcoma cells were in clear fusiform or polygonal form. The various nuclear forms were large and strongly stained with marked nucleoli (Fig. 1A and B). MyD88 staining in osteosarcoma tissues was mainly located in the cytoplasm. Positive staining was evident as a pale yellow, tan or brown color, representative of weak, moderate or strong staining, respectively. There were relatively fewer MyD88-positive cells in normal peritumoral bone tissues compared with osteosarcoma tissues (Fig. 1C and D). Statistical analysis demonstrated that the positive rate of MyD88 staining in osteosarcoma tissues was significantly increased (71.4\%) compared with that in matched normal peritumoral bone tissues $(6.1 \%$; $\mathrm{P}<0.05$; Table I).

Association between MyD88 protein and pathological characteristics and grading of patients with osteosarcoma. Statistical analysis of the clinicopathological status of patients with osteosarcoma in association with MyD88 protein revealed that MyD88 protein was not associated with gender, age, tumor site or subtype of patients with osteosarcoma $(\mathrm{P}>0.05)$. MyD88 positive expression is associated with metastasis and Enneking stage (both $\mathrm{P}<0.05)$ (Table II). 
Table I. Analysis of MyD88 expression in osteosarcoma and matched normal peritumoral bone tissues.

\begin{tabular}{lcccr}
\hline & & \multicolumn{2}{c}{ MyD88 expression } & \\
\cline { 3 - 4 } Tissue type & Total, $\mathrm{n}$ & Positive, $\mathrm{n}(\%)$ & Negative, $\mathrm{n}(\%)$ & P-value \\
\hline Osteosarcoma tissues & 98 & $70(71.4)$ & $28(28.6)$ & $<0.001^{\text {a }}$ \\
Adjacent non-tumor tissues & 98 & $6(6.1)$ & $91(92.9)$ & \\
\hline
\end{tabular}

${ }^{\mathrm{a}} \mathrm{P}<0.05$. MyD88, myeloid differentiation factor 88 .

Table II. Association between MyD88 and clinicopathological factors of patients with osteosarcoma (n=98).

\begin{tabular}{|c|c|c|c|c|}
\hline \multirow[b]{2}{*}{ Factor } & \multirow[b]{2}{*}{ Total, $\mathrm{n}$} & \multicolumn{2}{|c|}{ MyD88 expression } & \multirow[b]{2}{*}{ P-value } \\
\hline & & Positive, $\mathrm{n}$ & Negative, $\mathrm{n}$ & \\
\hline Sex & & & & 0.08 \\
\hline Male & 64 & 42 & 22 & \\
\hline Female & 34 & 28 & 6 & \\
\hline Age, years & & & & 0.22 \\
\hline$<18$ & 72 & 49 & 23 & \\
\hline$\geq 18$ & 26 & 21 & 5 & \\
\hline Anatomical site & & & & 0.74 \\
\hline Femur & 49 & 36 & 13 & \\
\hline Tibia & 42 & 30 & 12 & \\
\hline Other & 7 & 4 & 3 & \\
\hline Histological subtype & & & & 0.37 \\
\hline Osteoblastic & 63 & 48 & 15 & \\
\hline Chondroblastic & 24 & 15 & 9 & \\
\hline Others & 11 & 7 & 4 & \\
\hline Metastasis & & & & $<0.001^{\mathrm{a}}$ \\
\hline Yes & 43 & 40 & 3 & \\
\hline No & 55 & 30 & 25 & \\
\hline Enneking grade & & & & $<0.001^{\mathrm{a}}$ \\
\hline I & 10 & 2 & 8 & \\
\hline IIa & 24 & 12 & 12 & \\
\hline $\mathrm{IIb}$ & 53 & 46 & 7 & \\
\hline III & 11 & 10 & 1 & \\
\hline
\end{tabular}

${ }^{\mathrm{a}} \mathrm{P}<0.05$. MyD88, MyD88, myeloid differentiation factor 88 .

Association between MyD88 protein expression and clinical prognosis of patients with osteosarcoma. The Kaplan-Meier method was used to analyze the survival rate of post-surgery patients with osteosarcoma, with positive or negative MyD88 expression (Fig. 2). For patients with osteosarcoma with positive MyD88 expression, 26 cases succumbed within 5 years, the median survival time was 28.5 months and the overall 5 -year survival rate was $62.9 \%$. For osteosarcoma patients with negative MyD88 expression, 4 cases succumbed within 5 years, the median survival time was 52 months and the overall 5 -year survival rate was $85.7 \%$. The 5 -year survival rate was significantly reduced in patients with osteosarcoma displaying positive MyD88 expression compared with that in patients with negative MyD88 expression ( $\mathrm{P}=0.023)$ (Fig. 2).

Effects of ST2825 on the proliferation of osteosarcoma cells. The proliferation rate of osteosarcoma U2OS cells was analyzed in the presence of the MyD88 selective inhibitor, ST2825 (15 or $30 \mu \mathrm{M})$. A significant inhibition of the proliferation of osteosarcoma cells was demonstrated at $72 \mathrm{~h}$ of culture compared with that in the blank control and vehicle groups, in a dose-dependent manner ( $\mathrm{P}<0.05$; Fig. 3). The inhibition rate was significantly increased in the high-dose ST2825 treatment group compared with that in the low-dose treatment group at each time $(\mathrm{P}<0.05)$. 


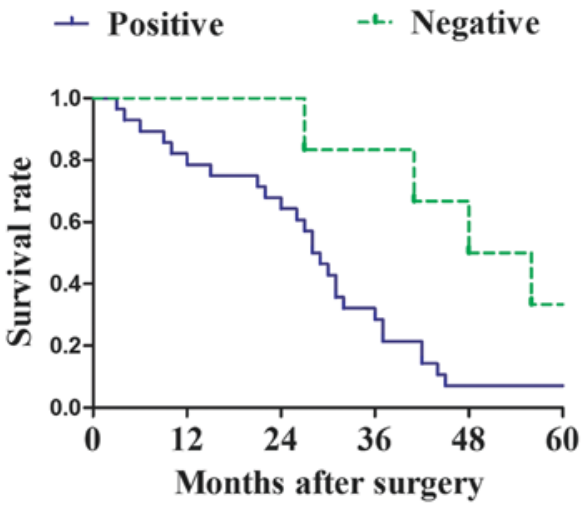

Figure 2. Kaplan-Meier curve analysis of MyD88 protein expression levels and the 5-year survival rate. The 5-year survival rate was significantly higher in patients with osteosarcoma with negative MyD88 expression compared with that in patients with positive MyD88 expression. MyD88, myeloid differentiation factor 88

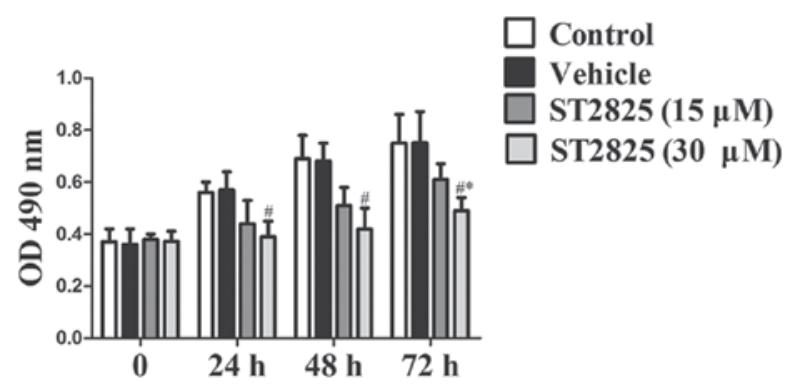

Figure 3. Effects of ST2825 on the proliferation rate of osteosarcoma U2OS cells in vitro. ST2825 significantly inhibited the proliferation of osteosarcoma cells in a dose-dependent manner. ${ }^{\# P}<0.05$ vs. ST2825 $(15 \mu \mathrm{M}) ;{ }^{*} \mathrm{P}<0.05$ vs. vehicle. OD, optical density.

Effects of ST2825 on the apoptosis of osteosarcoma cells. As shown in Fig. 4, the apoptosis rate of the osteosarcoma cells was unchanged between the solvent treatment group and the blank control group ( $\mathrm{P}>0.05)$. In response to ST2825 treatment $(15$ or $30 \mu \mathrm{M})$, there was a significant dose-dependent increase in the apoptotic rate of osteosarcoma cells compared with that of the control groups $(\mathrm{P}<0.05)$. In addition, the apoptosis rate was significantly increased in the high-dose treatment group compared with that in the low-dose treatment group $(\mathrm{P}<0.05)$.

ST2825 inhibits osteosarcoma cell migration in vitro. A Transwell migration assay was performed to detect the effects of ST2825 treatment on osteosarcoma cell migration. The effects of ST2825 treatment (15 or $30 \mu \mathrm{M})$ significantly decreased the number of osteosarcoma cells that migrated in the Transwell assay in a dose-dependent manner compared with that of the control or vehicle-treated group $(\mathrm{P}<0.05$; Fig. 5). In addition, the inhibitory effect of ST2825 treatment on osteosarcoma cell migration was significantly increased in the high-dose treatment group compared with that in the low-dose treatment group $(\mathrm{P}<0.05)$.

Effects of ST2825 on p65, cyclin D1, matrix-metallopeptidase-9 (MMP-9) and cleaved-caspase-3 protein expression in osteosarcoma cells. Given that high-dose ST2825 treatment was confirmed in the present study to produce an enhanced

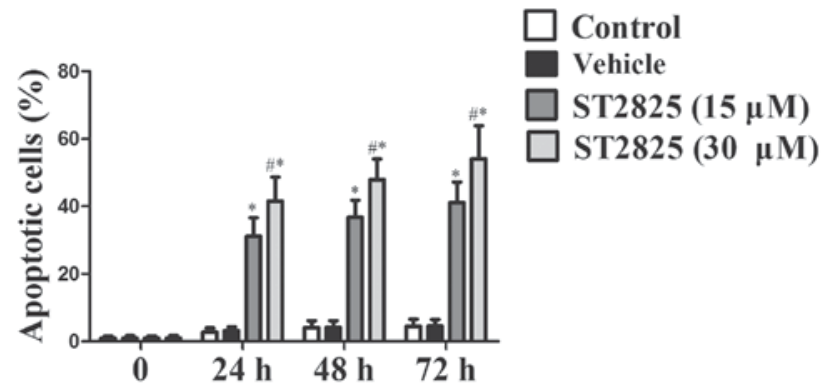

Figure 4. Effects of ST2825 on the apoptosis of osteosarcoma U2OS cells. ST2825 treatment significantly increased the apoptotic rate of osteosarcoma cells in a dose-dependent manner. The apoptosis rate was significantly higher in the high-dose treatment group compared with that in the low-dose treatment group. ${ }^{*} \mathrm{P}<0.05$ vs. ST2825 $(15 \mu \mathrm{M})$; ${ }^{*} \mathrm{P}<0.05$ vs. vehicle.

anti-proliferative and pro-apoptotic effect, this dose was used in subsequent experiments to study the potential mechanisms that may be involved. The results presented in Fig. 6 demonstrated that MMP-9, cyclin D1 and nuclear p65 expression were significantly decreased in osteosarcoma cells in the high-dose ST2825 treatment group compared with the control group, whilst cleaved-caspase 3 protein expression was significantly increased $(\mathrm{P}<0.05)$.

\section{Discussion}

The results from the present study demonstrated that an abnormally high level of MyD88 protein was apparent in human osteosarcoma cells. However, MyD88 expression was not associated with the gender, age, tumor site or subtype of patients with osteosarcoma ( $\mathrm{P}>0.05)$, yet the presence of osteosarcoma metastasis was associated with Enneking stage. In addition, the 5-year survival rate of patients with osteosarcoma with positive MyD88 expression was significantly lower compared with that of patients with negative MyD88 expression. Furthermore, the effects of ST2825, a selective inhibitor of MyD88, significantly inhibited the proliferation and promoted the apoptosis of osteosarcoma cells in vitro. The underlying mechanisms may involve inhibition of the nuclear transfer of NF- $\mathrm{BB}$ and p65, inhibition of Cyclin D1 and MMP9, and enhanced cleaved-caspase 3 protein expression. The results from the present study indicated that aberrant MyD88 expression may contribute to the development of osteosarcoma, and may thus be a potential novel therapeutic target for the treatment of this disease.

Previous studies have revealed abnormally high MyD88 expression in multiple types of tumor tissue, and the inhibition of MyD88 expression has been confirmed to be associated with the occurrence and development of various tumors (15). In the present study, inhibition of MyD88 significantly inhibited the proliferation of tumor cells and promoted apoptosis, indicating that MyD88 has potential as a novel target for tumor treatment $(16,17)$. Previous studies have confirmed that MyD88 is located in activated key nodes of multiple signal transduction pathways, and that activated MyD88 can regulate the activity of mitogen-activated protein kinases (MAPK) signaling pathways $(18,19)$. In addition, MyD88 can activate I $\kappa B$ kinase polymerase chain reaction, and further degrade IкB $\alpha$. Next NF- $\kappa B p 65$, which has been freed from I $\mathrm{kB} \alpha$, transfers from 
A

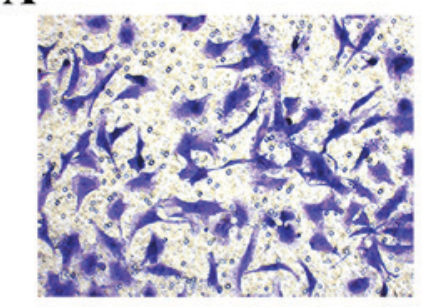

C

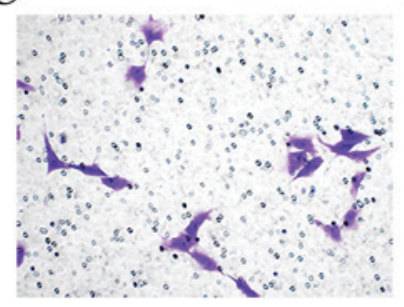

B

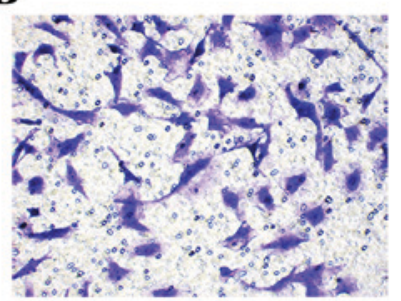

$\mathbf{D}$

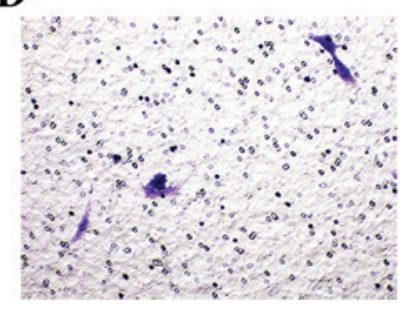

$\mathbf{E}$

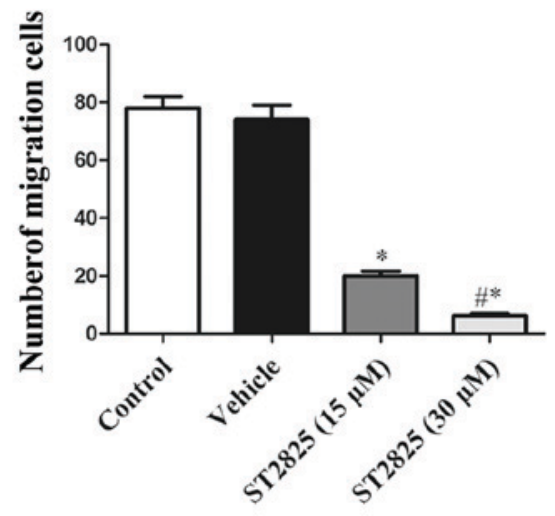

Figure 5. Effects of ST2825 on the migration of U2OS cells in vitro. Compared with (A) control or (B) vehicle, ST2825 at (C) $15 \mu \mathrm{M}$ or (D) $30 \mu \mathrm{M}$ significantly inhibited the migration of osteosarcoma U2OS cells in a dose-dependent manner (x400 magnification). (E) Quantification data for (A-D), " $\mathrm{P}<0.05$ vs. ST2825 $(15 \mu \mathrm{M}) ;{ }^{*} \mathrm{P}<0.05$ vs. vehicle.

A
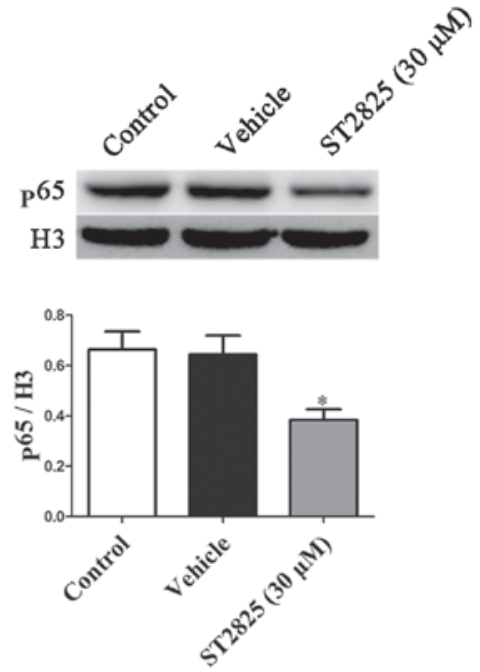

C
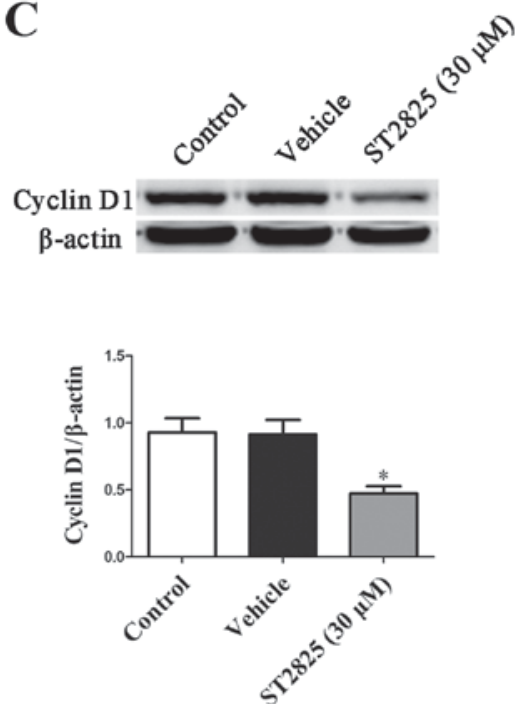

B

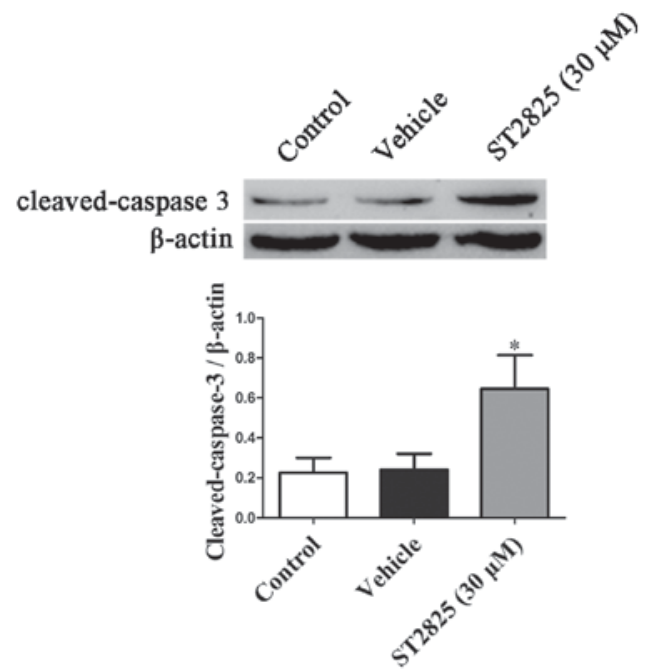

D
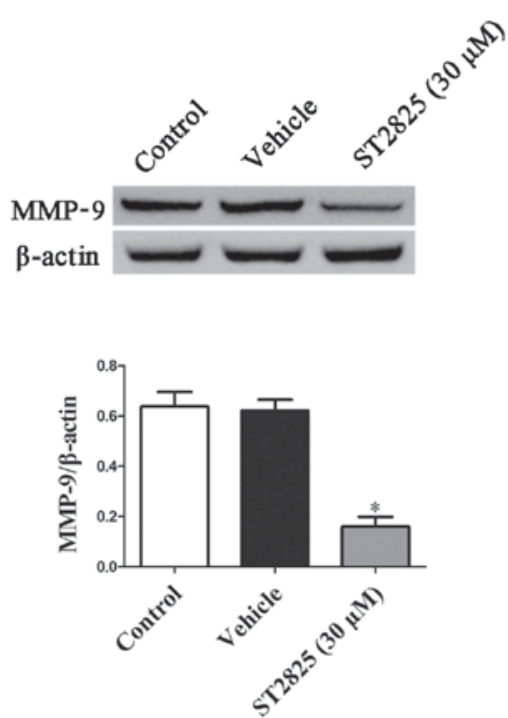

Figure 6. Western blot analysis of (A) p65, (B) cleaved-caspase 3, (C) cyclin D1 and (D) MMP-9 in high-dose ST2825-treated cells. High-dose ST2825 significantly inhibited nuclear p65 and cyclin D1 expression in osteosarcoma cells, and significantly increased MMP9 and cleaved-caspase 3 protein expression. ${ }^{*} \mathrm{P}<0.05$ vs. vehicle. MMP-9, matrix metallopeptidase-9. 
the cytoplasm to the nucleus (20). In turn, NF- $\kappa$ Bp65 binds to the specific downstream target gene sequence, thus regulating the transcription of associated-target genes. Previous studies have confirmed that MAPK expression is elevated in osteosarcoma tissues, and that inhibition of MAPK expression significantly inhibited the proliferation of osteosarcoma cells and promoted their apoptosis $(21,22)$. NF- $\mathrm{B}$ was also revealed to be abnormally activated in osteosarcoma tissues (23), and inhibition of $\mathrm{NF}-\kappa \mathrm{B}$ activity was shown to significantly inhibit the proliferation and promote the apoptosis of osteosarcoma cells (23-25). This suggests that MyD88 likely contributes an important function in the occurrence and development of osteosarcoma. Immunohistochemical analysis was adopted in the present study to investigate the MyD88 protein expression in the tumor tissues of patients with osteosarcoma. The results from the present study demonstrated that MyD88 expression was significantly higher in osteosarcoma tissues compared with that in normal peritumoral bone tissues. Further investigation revealed that MyD88 expression was not associated with gender, age or tumor sites of patients with osteosarcoma, but that it was associated with the presence or absence of osteosarcoma metastasis and Enneking stage. This is indicative of an important association of aberrant MyD88 expression in the development of osteosarcoma. The Kaplan-Meier method was used for statistical analysis of the survival rate of patients with osteosarcoma. The results from the present study revealed that the 5-year survival rate was significantly lower in patients with osteosarcoma with positive MyD88 expression compared with that in osteosarcoma patients with negative MyD88 expression, thus the promotion of MyD88 expression may be an important factor affecting the prognosis of osteosarcoma. Postoperative detection of the MyD88 expression in osteosarcoma tissues may be of particular importance in the prognosis of patients with osteosarcoma.

Following confirmation of the association between high expression of MyD88 in osteosarcoma tissues and patient prognosis, a specific inhibitor of MyD88, ST2825 was used to treat U2OS osteosarcoma cells during in vitro cell culture in the present study, to further investigate the function of MyD88 on the proliferation and apoptosis of osteosarcoma cells. The doses of ST2825 adopted in the present study were used as described in a previous study (11). The results of the present study demonstrated that ST2825 significantly inhibited the proliferation and promoted the apoptosis of osteosarcoma cells in a dose-dependent manner. The study revealed that $\mathrm{p} 65$ and cyclin $\mathrm{D} 1$ expression in the nuclei of osteosarcoma cells was significantly decreased in the ST2825 treatment group compared with that in the control group, indicating that this may be a mechanism of ST2825 inhibition of tumor cell proliferation. Cleaved-caspase 3 is the primary protein of apoptosis (25). In the present study, cleaved-caspase 3 protein was significantly increased in the ST2825 intervention group compared with the solvent group, suggesting that this may be a mechanism by which ST2825 inhibits the proliferation of osteosarcoma cells and promotes apoptosis. As, this effect of the selective inhibitor of MyD88 was only confirmed in the in vitro experiments, further in vivo studies are required in order to lay a solid theoretical basis for the treatment of osteosarcoma by targeting MyD88.
In conclusion, MyD88 expression is significantly increased in osteosarcoma tissues, and is significantly associated with clinical stage and prognosis. ST2825, a specific inhibitor of MyD88, inhibited the proliferation and promoted the apoptosis of osteosarcoma cells in vitro, suggesting that MyD88 may be a novel target for the treatment of osteosarcoma.

\section{Competing interests}

The authors declare that they have no competing interests.

\section{References}

1. Bishop MW, Janeway KA and Gorlick R: Future directions in the treatment of osteosarcoma. Curr Opin Pediatr 28: 26-33, 2016.

2. Ferrari S and Serra M: An update on chemotherapy for osteosarcoma. Expert Opin Pharmacother 16: 2727-2736, 2015.

3. Xiang F, Ni Z, Zhan Y, Kong Q, Xu J, Jiang J, Wu R and Kang X: Increased expression of MyD88 and association with paclitaxel resistance in breast cancer. Tumour Biol 37: 6017-6025, 2016.

4. Zhu Y, Huang JM, Zhang GN, Zha X and Deng BF: Prognostic significance of MyD88 expression by human epithelial ovarian carcinoma cells. J Transl Med 10: 77, 2012.

5. Liang B, Chen R, Wang T, Cao L, Liu Y, Yin F, Zhu M, Fan X, Liang Y, Zhang L, et al: Myeloid differentiation factor 88 promotes growth and metastasis of human hepatocellular carcinoma. Clin Cancer Res 19: 2905-2916, 2013.

6. Salcedo R, Worschech A, Cardone M, Jones Y, Gyulai Z, Dai RM, Wang E, Ma W, Haines D, O'HUigin C, et al: MyD88-mediated signaling prevents development of adenocarcinomas of the colon: Role of interleukin 18. J Exp Med 207: 1625-1636, 2010.

7. Kuo AH and Scheeren FA: Cell-intrinsic TLR2/MyD88 pathway in breast and colon cancer. Cell Cycle 13: 3785-3786, 2014.

8. Di Padova F, Quesniaux VFJ and Ryffel B: MyD88 as a therapeutic target for inflammatory lung diseases. Expert Opin Ther Targets 22: 401-408, 2018.

9. Tang QL, Xie XB, Wang J, Chen Q, Han AJ, Zou CY, Yin JQ, Liu DW, Liang Y, Zhao ZQ, et al: Glycogen synthase kinase-3 $\beta$, NF- $\mathrm{kB}$ signaling, and tumorigenesis of human osteosarcoma. J Natl Cancer Inst 104: 749-763, 2012.

10. Xu HY, Fang W, Huang ZW, Lu JC, Wang YQ, Tang QL, Song GH, Kang Y, Zhu XJ, Zou CY, et al: Metformin reduces SATB2-mediated osteosarcoma stem cell-like phenotype and tumor growth via inhibition of N-cadherin/NF-kB signaling. Eur Rev Med Pharmacol Sci 21: 4516-4528, 2017.

11. Lu Y, Li F, Xu T and Sun J: Tetrandrine prevents multidrug resistance in the osteosarcoma cell line, U-2OS, by preventing Pgp overexpression through the inhibition of $N F-\kappa B$ signaling. Int $J$ Mol Med 39: 993-1000, 2017.

12. Loiarro M, Capolunghi F, Fantò N, Gallo G, Campo S, Arseni B, Carsetti R, Carminati P, De Santis R, Ruggiero V and Sette C: Pivotal Advance: Inhibition of MyD88 dimerization and recruitment of IRAK1 and IRAK4 by a novel peptidomimetic compound. J Leukoc Biol 82: 801-810, 2017.

13. Zhang Y, Meng W and Cui H: LncRNA CBR3-AS1 predicts unfavorable prognosis and promotes tumorigenesis in osteosarcoma. Biomed Pharmacother 102: 169-174, 2018.

14. Zhou Y, Zhang W, Tang F, Luo Y, Min L, Zhang W, Shi R, Duan H and Tu C: A case report of apatinib in treating osteosarcoma with pulmonary metastases. Medicine (Baltimore) 96: e6578, 2017.

15. Chen X, Zhao F, Zhang H, Zhu Y, Wu K and Tan G: Significance of TLR4/MyD88 expression in breast cancer. Int J Clin Exp Pathol 8: 7034-7039, 2015.

16. Kfoury A, Virard F, Renno T and Coste I: Dual function of MyD88 in inflammation and oncogenesis: Implications for therapeutic intervention. Curr Opin Oncol 26: 86-91, 2014.

17. Deng Y, Sun J and Zhang LD: Effect of ST2825 on the proliferation and apoptosis of human hepatocellular carcinoma cells. Genet Mol Res 15: 15016826, 2016.

18. Shiratori E, Itoh M and Tohda S: MYD88 inhibitor ST2825 suppresses the growth of lymphoma and leukaemia cells. Anticancer Res 37: 6203-6209, 2017.

19. Warner N and Núñez G: MyD88: A critical adaptor protein in innate immunity signal transduction. J Immunol 190: 3-4, 2013.

20. Cervantes JL: MyD88 in Mycobacterium tuberculosis infection. Med Microbiol Immunol 206: 187-193, 2017. 
21. Deguine J and Barton GM: MyD88: A central player in innate immune signaling. F1000Prime Rep 6: 97, 2014.

22. Chandhanayingyong C, Kim Y, Staples JR, Hahn C and Lee FY: MAPK/ERK signaling in osteosarcomas, ewing sarcomas and chondrosarcomas: Therapeutic implications and future directions. Sarcoma 2012: 404810, 2012.

23. Meng Q, Zheng M, Liu H, Song C, Zhang W, Yan J, Qin L and Liu X: TRAF6 regulates proliferation, apoptosis, and invasion of osteosarcoma cell. Mol Cell Biochem 371: 177-186, 2012.

24. Miwa S, Sugimoto N, Yamamoto N, Shirai T, Nishida H, Hayashi K, Kimura H, Takeuchi A, Igarashi K, Yachie A and Tsuchiya H: Caffeine induces apoptosis of osteosarcoma cells by inhibiting AKT/mTOR/S6K, NF- $\mathrm{kB}$ and MAPK pathways. Anticancer Res 32: 3643-3649, 2012.
25. Zhao Z, Wu MS, Zou C, Tang Q, Lu J, Liu D, Wu Y, Yin J, Xie X, Shen J, et al: Downregulation of MCT1 inhibits tumor growth, metastasis and enhances chemotherapeutic efficacy in osteosarcoma through regulation of the NF- $\kappa \mathrm{B}$ pathway. Cancer Lett 342: $150-158,2014$.

c) (ㅇ) $\Theta$ This work is licensed under a Creative Common EY NO ND Attribution-NonCommercial-NoDerivatives 4.0 International (CC BY-NC-ND 4.0) License. 